\title{
Evaluación de medidas morfométricas de tendones y ligamentos metacarpales palmares por ultrasonografía en caballos criollos colombianos
}

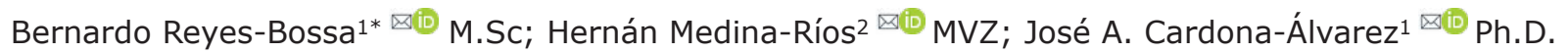

\begin{abstract}
${ }^{1}$ Universidad de Córdoba, Facultad de Medicina Veterinaria y Zootecnia, Departamento de Ciencias Pecuarias, Grupo de investigación en Medicina de Grandes Animales (MEGA). Montería, Colombia.

2Universidad de Antioquia, Facultad de Ciencias Agrarias, Grupo GAMMA. Medellín, Colombia.

* Correspondencia: breyesbossa@correo.unicordoba.edu.co
\end{abstract}

Recibido: Noviembre 2019; Aceptado: Mayo 2020; Publicado: Julio 2020.

\section{RESUMEN}

Objetivo. Determinar los parámetros morfométricos ultrasonográficos de los tendones y ligamentos metacarpales palmares de caballos criollos colombianos clínicamente sanos. Materiales y métodos. Treinta caballos criollos colombianos clínicamente sanos de $6.4 \pm 2.5$ años se evaluaron en diferentes zonas del departamento de Córdoba (Colombia). Se midieron las variables área transversal $\left(\mathrm{cm}^{2}\right)$, ancho latero medial $(A L M)(\mathrm{cm})$ y espesor dorso palmar $(E D P)(\mathrm{cm})$ en las estructuras de la región metacarpiana en los dos miembros anteriores mediante ultrasonografía. Resultados. La ecografía reveló que el área transversal del ligamento suspensorio fue la estructura de mayor área en las regiones proximales (1A, 1B y $2 \mathrm{~A}$ ), tanto para la extremidad izquierda como para la derecha. Por otro lado, no hubo una diferencia significativa $(p>0.05)$ entre la extremidad izquierda y derecha para las mediciones de las estructuras en cualquier zona, y no hubo una diferencia significativa entre los grupos de edades e índice de masa corporal (IMC) de los caballos. Conclusiones. Se obtuvieron valores de referencia para los tendones y ligamentos en la región metacarpiana palmar en equinos proporcionando una guía de referencia cuando se sospechan condiciones patológicas metacarpo en equinos.

Palabras clave: Caballos; anatomía; tendón; ligamento; metacarpo; extremidad anterior (Fuente: MeSH).

\section{ABSTRACT}

Objective. To determine the ultrasonographic morphometric parameters of the palmar metacarpal tendons and ligaments of clinically healthy Colombian creole horses. Materials and methods. Thirty clinically healthy Colombian creole horses of $6.4 \pm 2.5$ years were evaluated in different areas of the department of Córdoba (Colombia). The variables cross-sectional area $\left(\mathrm{cm}^{2}\right)$, medial lateral width $(A L M)(\mathrm{cm})$ and dorsal palmar thickness (EDP) $(\mathrm{cm})$ in the structures of the metacarpal region in the two anterior members were measured by means of ultrasound. Results. Ultrasonography revealed 
that the transverse area of the suspensory ligament was the structure with the largest area in the proximal regions ( $1 \mathrm{~A}, 1 \mathrm{~B}$ and $2 \mathrm{~A})$, for both the left and right limbs. On the other hand, there was no significant difference $(P>0.05)$ between left and right limb for measurements of structures in any area, and there was no significant difference between age groups and body mass index (BMI) of horses. Conclusions. Reference values for tendons and ligaments in the palmar metacarpal region were obtained in equines providing a reference guide when metacarpal pathological conditions in equines are suspected.

Keywords: Horses; anatomy; tendon; ligament; metacarpus; forelimb (Source: MeSH).

\section{INTRODUCCIÓN}

La ultrasonografía es una técnica médica que ha sido utilizada en los equinos con gran acogida, la cual fue introducida en 1982 por Rantanen (1). Esta técnica ayuda en el diagnóstico de lesiones en tejidos del organismo animal y humano, revelando el sitio, tamaño y grado de compromiso estructural, siendo necesaria para que el veterinario pueda diferenciar entre patrones ecográficos normales y anormales (2).

El examen de estructuras tendinosas y ligamentosas en caballos mediante el uso de ultrasonografía se ha convertido rápidamente en un procedimiento estándar, en donde es utilizada en el día a día de la practica equina para fines de diagnóstico y evaluación de alguna serie de lesiones o monitoreo de tratamientos (3).

Las cojeras en equinos ocasionadas por trastornos en los tejidos blandos suelen aparecer en las extremidades anteriores especialmente en los tendones y ligamentos del área metacarpiana palmar. El diagnóstico ecográfico ha demostrado ser seguro y no invasivo para detectar estas lesiones lo que permite su evaluación morfológica y que es más importante su observación a lo largo del tiempo (5).

Variaciones en el área transversal de ligamentos y tendones de la región metacarpal palmar en caballos (PSI, cuarto de milla, árabes, pura sangre español) clínicamente sanos han sido reportadas. De igual manera la influencia de la edad, sexo, miembro e índice de masa corporal (IMC) en los parámetros ecográficos como el área transversal de los tendones ha sido estudiado $(2,6,7)$.

En el caballo criollo colombiano existe poca evidencia reportada en relación con esta temática. Dado lo anterior el propósito de este estudio fue determinar los parámetros morfométricos ultrasonográficos de tendones y ligamentos metacarpales palmares de caballos criollos colombianos clínicamente sanos, y así mismo estudiar el efecto de la edad, sexo, miembro e IMC sobre estos parámetros morfológicos de estas estructuras en estos animales.

\section{MATERIALES Y MÉTODOS}

Lineamientos éticos. La manipulación y toma de medidas en los caballos estudiados fueron aprobabas por el Comité de Ética Animal de la Facultad de Medicina Veterinaria y Zootecnia de la Universidad de Córdoba (Colombia) (acta: 013 de marzo del 2022).

Animales. 30 caballos criollos colombianos clínicamente sanos fueron utilizados en el estudio, 10 machos y 20 hembras, con edades entre los 4 y 14 años la cual fue corroborada por cronometría dentaria (8), con un peso corporal entre 250 y $450 \mathrm{~kg}$, y una alzada entre 1.29 y 1.46 metros. El IMC se calculó como: (Peso $(\mathrm{kg})$ / [Alzada a la cruz $(\mathrm{m})]^{2}$ ) (6). Todos los animales del estudio se encontraban en edad de entrenamiento (9).

Se evaluó clínicamente (inspección, palpación y examen dinámico) los miembros anteriores de los individuos en estudio, con criterios de inclusión de: animales que distribuyeran el peso en los cuatro miembros, caballos sin cojera, a la inspección no presentaran agrandamiento de tendones o ligamentos y sin respuestas dolorosas a la palpación de tendones o ligamentos $(2,10)$.

Evaluación ultrasonográfica. La preparación de los animales para el examen ultrasonográfico se realizó de la siguiente manera: el pelo de la región comprendida entre el aspecto distal de hueso accesorio del carpo (Os carpis accesorium) $y$ el aspecto proximal de los sesamoideos proximales (11), fue retirado en su totalidad con una máquina electrónica whall series pro (Whall, 2018, USA), al igual que en el aspecto medial y lateral por encima del menudillo. Luego, la región fue medida con una cinta métrica y dividida en 5 
partes iguales (7). A partir de los $4 \mathrm{~cm}$ por debajo del aspecto más palmar del Os carpis accesorium se delimitó las zonas con base al cálculo anterior y los puntos se señalaron con acuarela blanca en el aspecto lateral de la región. Seguido a esto se aplicó un lavado con jabón quirúrgico y agua tibia en la zona a estudiar y también se realizó un lavado con alcohol para desengrase de la piel $(4,11)$. Por último, se aplicó gel de ultrasonografía en el área a estudiar (Figura 1).

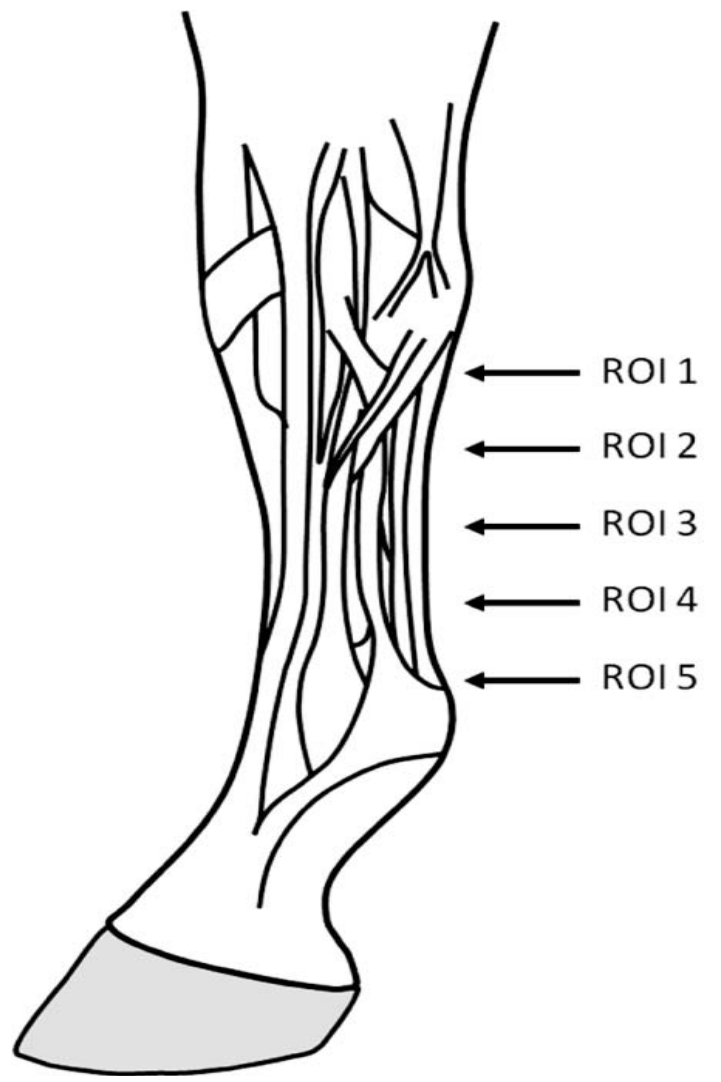

Figura 1. Esquema de la delimitación de las zonas del estudio (ROI: región de interés), tomado de Boehart et al (7).

En todos los animales la evaluación ultrasonográfica se realizó por el mismo operario para evitar las posibles variaciones interoperador (12). Se obtuvo imágenes ecográficas transversales (plano anatómico dorsal) de la región metacarpiana en el aspecto palmar (Zona 1A, 1B, 2A, 2B y 3A). El examen ecográfico se realizó con un equipo ultrasonográfico móvil (Mindray M6, 2018, USA) en combinación con una sonda lineal (Mindray) de 8 a $10 \mathrm{MHz}$ y stand off pad. Se tomaron dos imágenes de zona en cada miembro y en la última región fueron tomadas seis imágenes (palmar, medial y lateral). Las estructuras estudiadas de palmar a dorsal fueron el tendo musculi flexor digitalis superficialis (Tendon flexor digital superficial TFDS), tendo musculi flexor digitalis profundus (Tendon flexor digital profundo TFDP), ligamentum accessorium (Ligamento accesorio LA del TFDP) y el cuerpo del musculus interosseus medius (Ligamento suspensorio LS), y también las ramas medial y lateral del LS (6).

Configuraciones de enfoque, brillo, contraste y ganancia constantes se utilizaron durante las sesiones de ultrasonografía, siendo las mismas para todos los tendones y ligamentos en estudio (3).

Toma de mediciones. De las imágenes obtenidas se realizaron las mediciones utilizando el software de medición del equipo. Las siguientes variables fueron medidas para cada estructura en cada zona: área transversal (AT) $\left(\mathrm{cm}^{2}\right)$, ancho latero medial (LM) y espesor dorso palmar (DP) medidas en centímetros. El área transversal se obtuvo delimitando los bordes de cada estructura, el LM desde el punto más lateral hasta el más medial de cada estructura y el DP desde el punto más dorsal hasta el más palmar en todas las zonas por igual. El valor final para cada variable fue la media entre las dos imágenes que se tomaron para cada estructura; si el coeficiente de variación (CV) era $>10 \%$, se realizaban dos nuevas mediciones (3).

Todas las evaluaciones ultrasonográficas se realizaron de noche, con el animal distribuyendo su peso en los 4 miembros. Los animales fueron sedados con xilacina al $10 \%$ (Erma, xilacina $10 \%$, 2019, Colombia), a dosis de $1 \mathrm{mg} / \mathrm{kg}$, usando una jeringa desechable de $5 \mathrm{ml}$ y agujas de 18 Gauges por animal (13).

Recolección de datos. Todos los datos fueron tabulados en una hoja electrónica (Microsoft Excel, 2010), y se realizó la estadística descriptiva. Se validaron los supuestos de normalidad y homogeneidad de varianza. Se realizó un modelo mixto lineal de 4 factores donde se incluyó el "animal" como factor aleatorio y "estructura", "medida" y "zona" como factores fijos. En relación con la edad, se formaron 3 grupos: grupo $1(n=13)$ incluía caballos $\leq 5$ años, grupo $2(n=12)$ incluía caballos de 5 a 10 años y grupo $3(n=5)$ incluía caballos $\geq 10$ años. Para el sexo se formaron 2 grupos: grupo $1(n=13)$ incluía sementales y castrados y grupo $2(n=17)$ incluía yeguas. En el IMC se formaron 2 grupos: grupo $1(n=7)$ incluía caballos con un IMC $\leq 178$ $\mathrm{kg} / \mathrm{m}^{2}$ y grupo $2(\mathrm{n}=23)$ incluía caballos con un IMC> $178 \mathrm{~kg} / \mathrm{m}^{2}$. 
El análisis de varianza de una vía (para edad) se utilizó para determinar la influencia de las diferentes variables en el tamaño del tendón y la prueba t de student (para sexo, IMC y miembro) para cada combinación de zonas y estructuras. Se observaron las diferencias entre las zonas y las diferencias entre las estructuras, pero no se encontraron diferencias sustanciales entre los otros subgrupos. Para todas las pruebas, un valor de $p \leq 0.05$ se consideró significativo. Todos los análisis se realizaron en el software R-Project.

\section{RESULTADOS}

Los valores de las mediciones morfométricas de los tendones flexores y los ligamentos metacarpales de los animales del estudio se describen en la tabla 1. Las estructuras observadas en la ecografía se pueden apreciar en la figura 2, así como en las figuras 3-6 que se describe el comportamiento de las medidas de los tendones y ligamentos estudiados en cada zona según las variables en los 30 caballos del estudio.

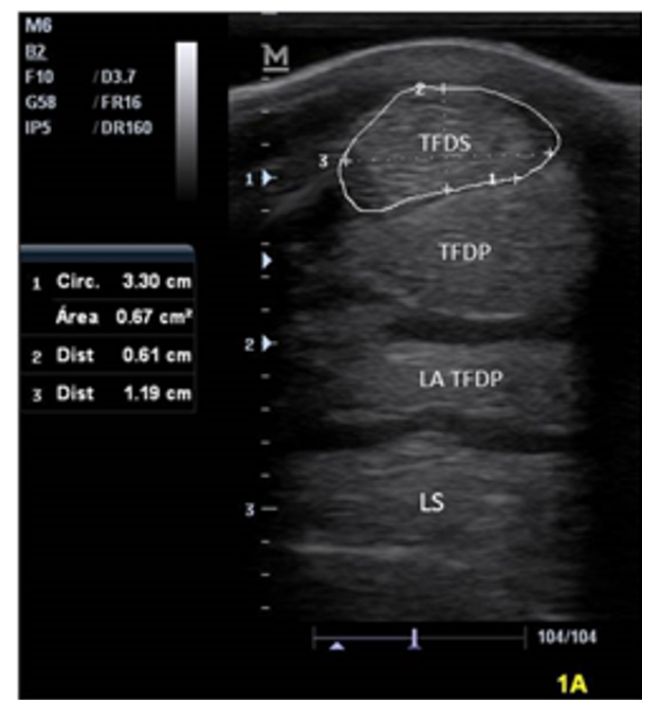

Figura 2. Imagen ecográfica de la zona $1 \mathrm{~A}$ del aspecto palmar del metacarpo de un equino criollo colombiano (TFDS: tendón flexor digital superficial, TFDP: tendón flexor digital profundo, LA: ligamento accesorio, LS: ligamento suspensorio).
Tabla 1. Valores medios $\pm D E$ de las mediciones morfométricas ecográficas de tendones flexores digitales y ligamentos palmares de la región metacarpal en 5 zonas.

\begin{tabular}{|c|c|c|c|c|c|c|c|}
\hline \multirow[b]{2}{*}{ Est } & \multirow[b]{2}{*}{ Zona } & \multicolumn{3}{|c|}{ Miembro Izquierdo } & \multicolumn{3}{|c|}{ Miembro Derecho } \\
\hline & & $\underset{\left(\mathrm{cm}^{2}\right)}{\mathbf{A T}}$ & $\begin{array}{c}\text { LM } \\
(\mathbf{c m})\end{array}$ & $\begin{array}{c}\text { DP } \\
(\mathbf{c m})\end{array}$ & AT & LM & DP \\
\hline \multirow{5}{*}{ TFDS } & $1 \mathrm{~A}$ & $\begin{array}{c}0.518 \\
\pm 0.086\end{array}$ & $\begin{array}{c}1.054 \\
\pm 0.098\end{array}$ & $\begin{array}{c}0.519 \\
\pm 0.072\end{array}$ & $\begin{array}{c}0.507 \\
\pm 0.085\end{array}$ & $\begin{array}{c}1.042 \\
\pm 0.101\end{array}$ & $\begin{array}{c}0.512 \\
\pm 0.075\end{array}$ \\
\hline & $1 \mathrm{~B}$ & $\begin{array}{c}0.494 \\
\pm 0.067\end{array}$ & $\begin{array}{c}1.123 \\
\pm 0.099\end{array}$ & $\begin{array}{c}0.432 \\
\pm 0.061\end{array}$ & $\begin{array}{c}0.467 \\
\pm 0.074\end{array}$ & $\begin{array}{c}1.091 \\
\pm 0.114\end{array}$ & $\begin{array}{c}0.427 \\
40.043\end{array}$ \\
\hline & $2 A$ & $\begin{array}{c}0.503 \\
\pm 0.089\end{array}$ & $\begin{array}{c}1.185 \\
\pm 0.113\end{array}$ & $\begin{array}{c}0.377 \\
\pm 0.055\end{array}$ & $\begin{array}{c}0.499 \\
\pm 0.089\end{array}$ & $\begin{array}{c}1.203 \\
\pm 0.132\end{array}$ & $\begin{array}{c}0.372 \\
\pm 0.055\end{array}$ \\
\hline & $2 B$ & $\begin{array}{c}0.556 \\
\pm 0.092\end{array}$ & $\begin{array}{c}1.335 \\
\pm 0.112\end{array}$ & $\begin{array}{c}0.364 \\
\pm 0.060\end{array}$ & $\begin{array}{c}0.536 \\
\pm 0.087\end{array}$ & $\begin{array}{c}1.344 \\
\pm 0.139\end{array}$ & $\begin{array}{c}0.341 \\
\pm 0.043\end{array}$ \\
\hline & $3 A$ & $\begin{array}{c}0.602 \\
\pm 0.085\end{array}$ & $\begin{array}{c}1.693 \\
\pm 0.157\end{array}$ & $\begin{array}{c}0.293 \\
\pm 0.037\end{array}$ & $\begin{array}{c}0.609 \\
\pm 0.093\end{array}$ & $\begin{array}{c}1.655 \\
\pm 0.215\end{array}$ & $\begin{array}{c}0.296 \\
\pm 0.046\end{array}$ \\
\hline \multirow{5}{*}{ TFDP } & $1 \mathrm{~A}$ & $\begin{array}{c}0.769 \\
\pm 0.121\end{array}$ & $\begin{array}{c}1.092 \\
\pm 0.112\end{array}$ & $\begin{array}{c}0.732 \\
2 \pm 0.071\end{array}$ & $\begin{array}{c}0.756 \\
\pm 0.108\end{array}$ & $\begin{array}{c}1.078 \\
\pm 0.101\end{array}$ & $\begin{array}{c}0.733 \\
1 \pm 0.086\end{array}$ \\
\hline & $1 \mathrm{~B}$ & $\begin{array}{c}0.617 \\
\pm 0.083\end{array}$ & $\begin{array}{c}0.947 \\
3 \pm 0.081\end{array}$ & $\begin{array}{c}0.718 \\
1 \pm 0.061\end{array}$ & $\begin{array}{c}0.642 \\
\pm 0.085\end{array}$ & $\begin{array}{c}0.955 \\
\pm 0.064\end{array}$ & $\begin{array}{r}0.725 \\
4 \pm 0.076\end{array}$ \\
\hline & $2 \mathrm{~A}$ & $\begin{array}{c}0.576 \\
\pm \\
0.053\end{array}$ & $\begin{array}{c}0.904 \\
\pm 0.076\end{array}$ & $\begin{array}{c}0.701 \\
\pm 0.062\end{array}$ & $\begin{array}{c}0.592 \\
\pm 0.068\end{array}$ & $\begin{array}{c}0.918 \\
\pm 0.082\end{array}$ & $\begin{array}{c}0.728 \\
2 \pm 0.075\end{array}$ \\
\hline & $2 B$ & $\begin{array}{c}0.626 \\
\pm 0.086\end{array}$ & $\begin{array}{c}1.021 \\
\pm 0.109\end{array}$ & $\begin{array}{c}0.688 \\
\pm 0.068\end{array}$ & $\begin{array}{c}0.638 \\
\pm 0.107\end{array}$ & $\begin{array}{c}1.024 \\
\pm 0.124\end{array}$ & $\begin{array}{c}0.701 \\
4 \pm 0.071\end{array}$ \\
\hline & $3 A$ & $\begin{array}{c}0.925 \\
\pm 0.112 \\
\end{array}$ & $\begin{array}{r}1.318 \\
- \pm 0.140 \\
\end{array}$ & $\begin{array}{c}0.793 \\
\pm 0.078 \\
\end{array}$ & $\begin{array}{c}0.956 \\
\pm 0.137\end{array}$ & $\begin{array}{c}1.269 \\
\pm 0.199 \\
\end{array}$ & $\begin{array}{c}0.833 \\
9 \pm 0.092\end{array}$ \\
\hline \multirow{4}{*}{ LA } & $1 \mathrm{~A}$ & $\begin{array}{c}0.535 \\
\pm 0.112\end{array}$ & $\begin{array}{c}1.054 \\
\pm 0.119\end{array}$ & $\begin{array}{c}0.436 \\
\pm 0.085\end{array}$ & $\begin{array}{c}0.498 \\
\pm 0.103\end{array}$ & $\begin{array}{c}1.022 \\
\pm 0.121\end{array}$ & $\begin{array}{c}0.414 \\
1 \pm 0.090\end{array}$ \\
\hline & $1 \mathrm{~B}$ & $\begin{array}{c}0.423 \\
\pm 0.082\end{array}$ & $\begin{array}{c}0.885 \\
\pm 0.110\end{array}$ & $\begin{array}{c}0.390 \\
\pm 0.059\end{array}$ & $\begin{array}{c}0.429 \\
\pm 0.093\end{array}$ & $\begin{array}{c}0.899 \\
\pm 0.134\end{array}$ & $\begin{array}{c}0.392 \\
4 \pm 0.072\end{array}$ \\
\hline & $2 \mathrm{~A}$ & $\begin{array}{c}0.379 \\
\pm 0.082\end{array}$ & $\begin{array}{c}0.818 \\
\pm 0.126\end{array}$ & $\begin{array}{c}0.336 \\
\pm 0.068\end{array}$ & $\begin{array}{c}0.371 \\
\pm 0.092\end{array}$ & $\begin{array}{c}0.828 \\
\pm 0.104\end{array}$ & $\begin{array}{c}0.355 \\
4 \pm 0.087\end{array}$ \\
\hline & $2 B$ & $\begin{array}{c}0.329 \\
\pm 0.066 \\
\end{array}$ & $\begin{array}{l}0.843 \\
\pm 0.10\end{array}$ & $\begin{array}{c}0.307 \\
\pm 0.049\end{array}$ & $\begin{array}{c}0.310 \\
\pm 0.062 \\
\end{array}$ & $\begin{array}{c}0.845 \\
\pm 0.095 \\
\end{array}$ & $\begin{array}{c}0.277 \\
\pm 0.053 \\
\end{array}$ \\
\hline \multirow{4}{*}{ LS } & $1 \mathrm{~A}$ & $\begin{array}{c}0.840 \\
\pm 0.133\end{array}$ & $\begin{array}{c}1.171 \\
3 \pm 0.118\end{array}$ & $\begin{array}{c}0.705 \\
3 \pm 0.069\end{array}$ & $\begin{array}{c}0.871 \\
\pm 0.134\end{array}$ & $\begin{array}{r}1.170 \\
+ \pm 0.114\end{array}$ & $\begin{array}{c}0.726 \\
4 \pm 0.064\end{array}$ \\
\hline & $1 \mathrm{~B}$ & $\begin{array}{c}0.826 \\
\pm 0.117\end{array}$ & 1.135 & 0.722 & $\begin{array}{c}0.834 \\
\pm 0.119\end{array}$ & $\begin{array}{c}1.144 \\
\pm 0.116\end{array}$ & $\begin{array}{c}0.725 \\
6 \pm 0.054\end{array}$ \\
\hline & $2 \mathrm{~A}$ & $\begin{array}{c}0.820 \\
\pm 0.118\end{array}$ & 1.104 & 0.721 & $\begin{array}{c}0.847 \\
\pm 0.116\end{array}$ & $\begin{array}{c}1.133 \\
\pm 0.115\end{array}$ & $\begin{array}{c}0.734 \\
5 \pm 0.063\end{array}$ \\
\hline & 2B & $\begin{array}{c}0.959 \\
\pm 0.188 \\
\end{array}$ & $\begin{array}{c}1.183 \\
3 \pm 0.146 \\
\end{array}$ & 0.780 & $\begin{array}{c}0.916 \\
\pm 0.150 \\
\end{array}$ & $\begin{array}{c}1.194 \\
\pm 0.121 \\
\end{array}$ & $\begin{array}{c}0.716 \\
1 \pm 0.157 \\
\end{array}$ \\
\hline RM & $3 A$ & $\begin{array}{c}0.631 \\
\pm 0.131\end{array}$ & $\begin{array}{c}0.638 \\
\pm 0.076\end{array}$ & $\begin{array}{c}1.011 \\
\pm 0.123\end{array}$ & $\begin{array}{c}0.630 \\
\pm 0.128\end{array}$ & $\begin{array}{c}0.621 \\
\pm 0.067\end{array}$ & $\begin{array}{c}1.027 \\
\pm 0.116\end{array}$ \\
\hline RL & $3 A$ & $\begin{array}{c}0.652 \\
\pm 0.159\end{array}$ & $\begin{array}{c}0.645 \\
\pm 0.085\end{array}$ & 1.026 & $\begin{array}{c}0.662 \\
\pm 0.089\end{array}$ & $\begin{array}{c}0.655 \\
\pm 0.048\end{array}$ & $\begin{array}{c}1.042 \\
8 \pm 0.088\end{array}$ \\
\hline
\end{tabular}

Est: Estructura; TFDS: tendón flexor digital superficial; TFDP: tendón flexor digital profundo; LA: ligamento accesorio; LS: ligamento suspensorio; RM: rama medial del ligamento suspensorio, RL: rama lateral del ligamento suspensorio; AT: área de la sección transversal; LM: ancho latero medial; DP: ancho dorso palmar. 


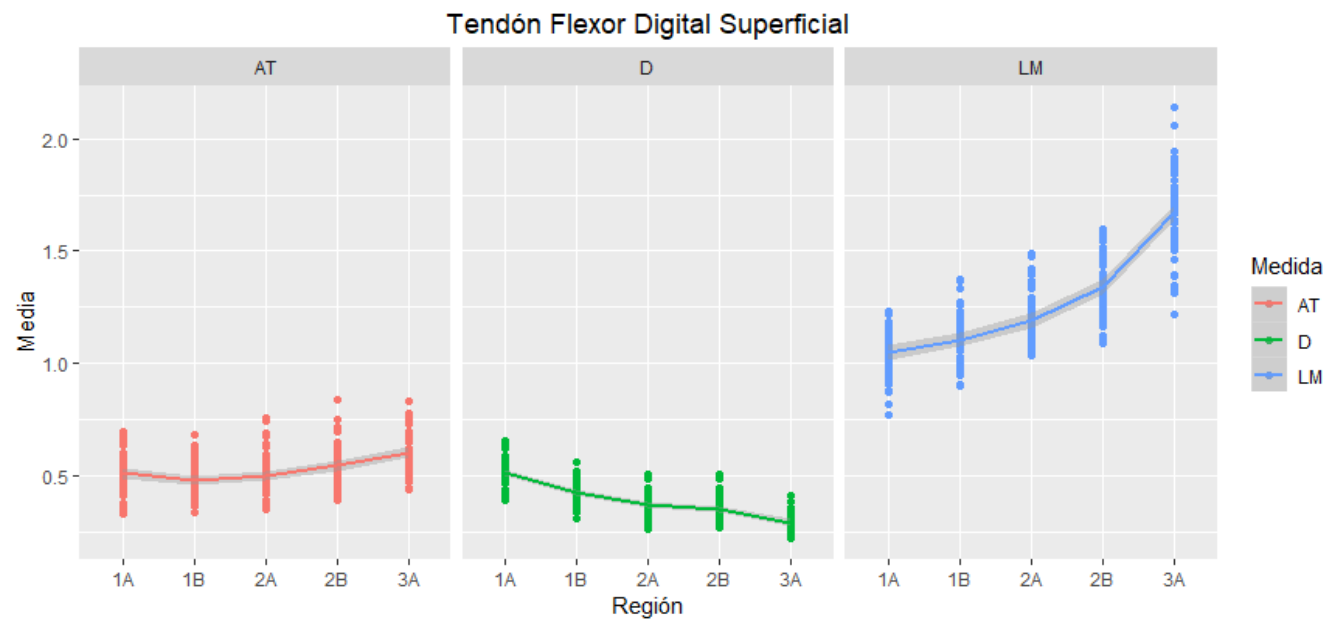

Figura 3. Media de los valores ecográficos del tendón flexor digital superficial de la región metacarpiana (1A3A) de 30 caballos criollos colombianos. AT: área transversal, D: espesor dorsopalmar y LM: ancho lateromedial.

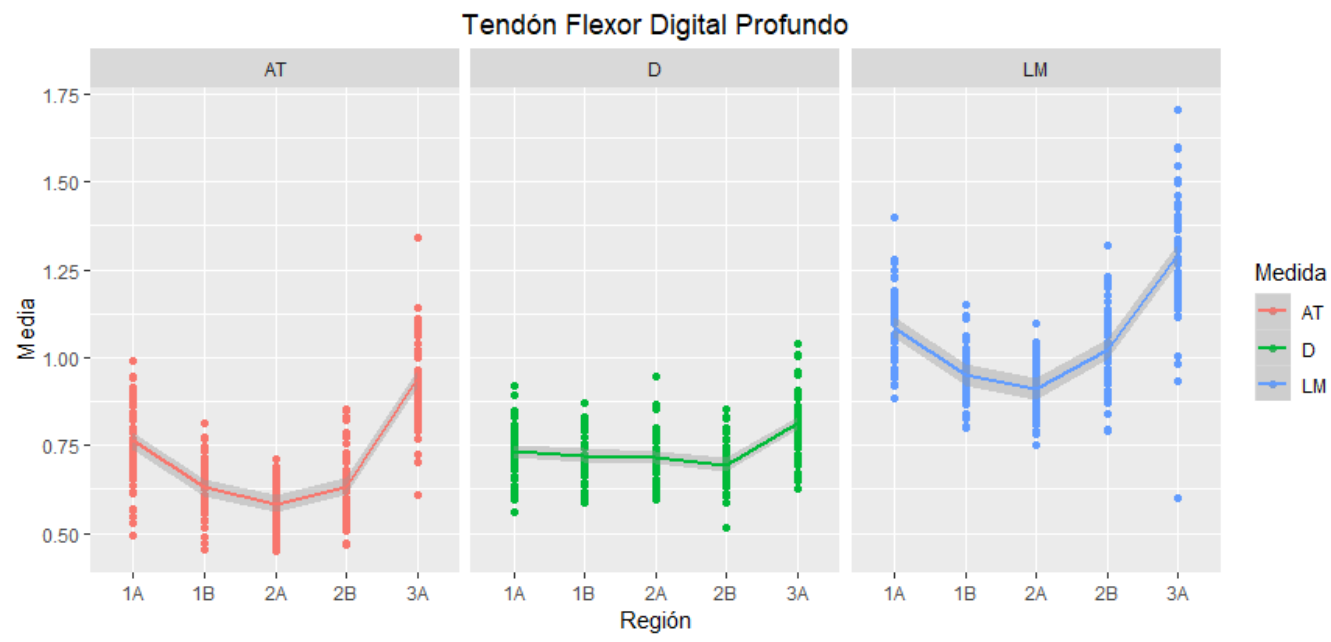

Figura 4. Media de los valores ecográficos del tendón flexor digital profundo de la región metacarpiana (1A3A) de 30 caballos criollos colombianos. AT: área transversal, D: espesor dorsopalmar y LM: ancho lateromedial.

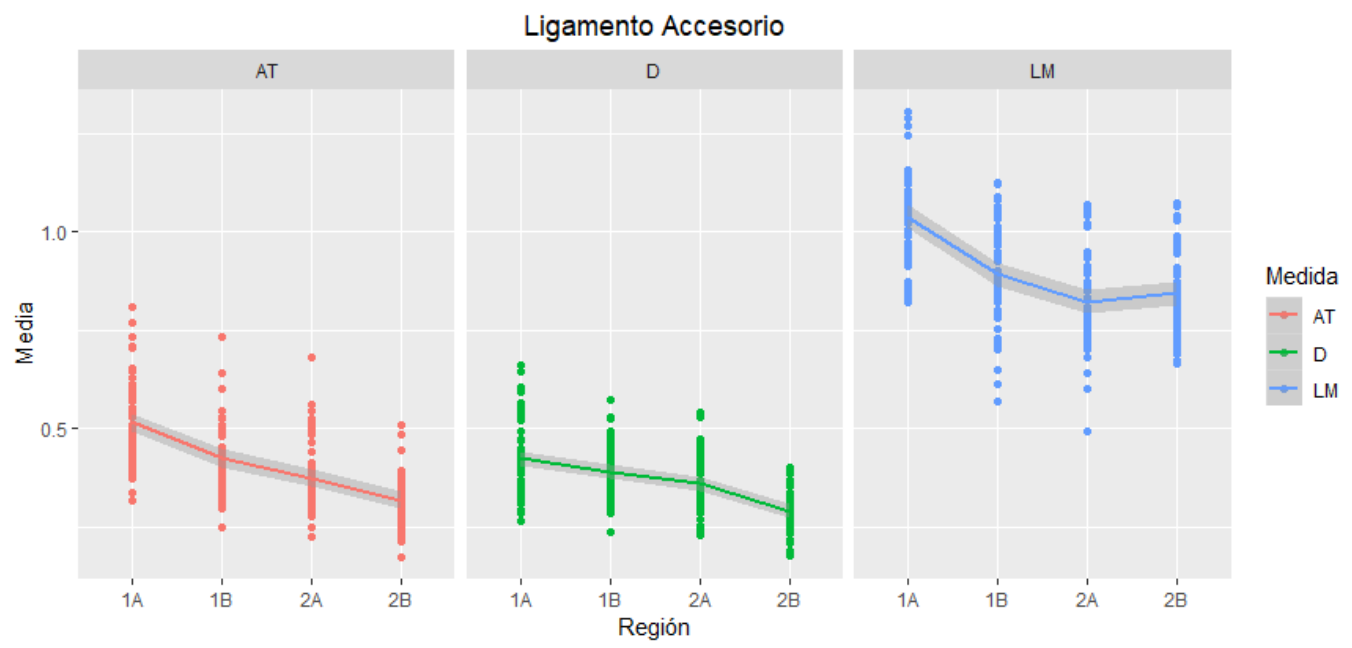

Figura 5. Media de los valores ecográficos del ligamento accesorio del TFDP de la región metacarpiana (1A2B) de 30 caballos criollos colombianos. AT: área transversal, D: espesor dorsopalmar y LM: ancho lateromedial. TFDP: Tendón flexor digital profundo. 


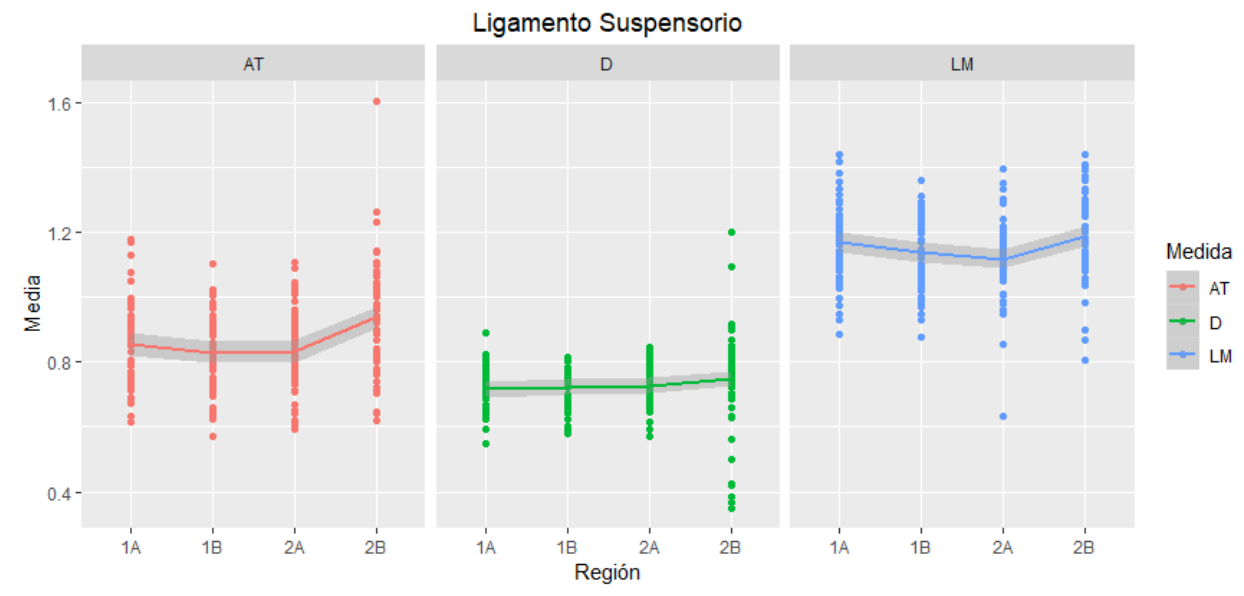

Figura 6. Media de los valores ecográficos del ligamento suspensorio de la región metacarpiana (1A-2B) de 30 caballos criollos colombianos. AT: área transversal, D: espesor dorsopalmar y LM: ancho lateromedial. Diferencias de las medidas de los tendones
y ligamentos metacarpales palmares
entre grupos. En los grupos conformados no se encontró diferencia significativa $(p>0.05)$ entre las mediciones del miembro izquierdo en comparación con el miembro derecho. De igual manera, no se encontró diferencia significativa $(p>0.05)$ entre los grupos de edades e IMC. Para el caso del sexo hubo diferencia significativa $(p<0.05)$ en el LM del TFDP en las zonas $1 \mathrm{~A}$ y $2 B(p=0.039$ y $p=0.033)$; al igual que el $A T$ del LA en las zonas $1 A$ y $2 A(p=0.012$ y $p=0,043)$ $y$ el LM del mismo en la zona $1 A(p=0.027)$.

Al evaluar cada zona, se encontró que existió diferencia entre las estructuras de la misma zona (Tabla 2).

En el LM, se encontró que en estos animales esta variable se comporta muy similar en el TFDS y LS en la región proximal, siendo las dos estructuras de mayor tamaño en este aspecto, seguido del TFDP y el LA del TFDP fue la estructura de menor tamaño en las regiones proximales. El TFDS en las zonas distales presentó valores más altos seguido del TFDP que ya se encuentra unido a su ligamento accesorio y la estructura de menor valor de LM fueron las ramas del LS.

\section{Diferencias de las estructuras en las diferentes} zonas. Para el caso del área transversal del tendón, en los caballos criollos colombianos del estudio, el ligamento suspensorio fue la estructura de mayor área en las regiones proximales (1A, 1B y $2 \mathrm{~A})$, seguido del TFDP, pero el TFDS presentó mayores valores que el Ligamento accesorio del TFDP. En las zonas distales ( $2 \mathrm{~B}$ y $3 \mathrm{~A}$ ), las estructuras con mayor área fueron el ligamento suspensorio (cuerpo y ramas) y el TFDP.
Tabla 2. Comparación (diferencia de medias) de mediciones morfométricas ultrasonográficas entre estructuras de los tendones flexores digitales y ligamentos de la región metacarpal palmar en 30 CCC.

\begin{tabular}{|c|c|c|c|c|c|}
\hline Zona & Est & EC & AT & LM & DP \\
\hline \multirow[t]{6}{*}{$1 \mathrm{~A}$} & TFDS & TFDP & $<0.001 * * *$ & 0.254 & $<0.001 * * *$ \\
\hline & & LA & 0.998 & 0.964 & $<0.001 * * *$ \\
\hline & & LS & $<0.001 * * *$ & $<0.001 * * *$ & 0.075 \\
\hline & TFDP & LA & $<0.001 * * *$ & 0.095 & $<0.001 * * *$ \\
\hline & & LS & $<0.001 * * *$ & $<0.001 * * *$ & 0.646 \\
\hline & LA & LS & $<0.001 * * *$ & $<0.001 * * *$ & $<0.001 * * *$ \\
\hline \multirow[t]{6}{*}{ 1B } & TDFS & TFDP & $<0.001 * * *$ & $<0.001 * * *$ & 0.165 \\
\hline & & LA & $0.006 * *$ & $<0.001 * * *$ & $<0.001 * * *$ \\
\hline & & LS & $<0.001 * * *$ & 0.337 & $0.037 *$ \\
\hline & TFDP & LA & $<0.001 * * *$ & $0.012 *$ & $<0.001 * * *$ \\
\hline & & LS & $<0.001 * * *$ & $<0.001 * * *$ & 0.865 \\
\hline & LA & LS & $<0.001 * * *$ & $<0.001 * * *$ & $<0.001 * * *$ \\
\hline \multirow[t]{6}{*}{$2 \mathrm{~A}$} & TFDS & TFDP & $<0.001 * * *$ & $<0.001 * * *$ & 0.859 \\
\hline & & LA & $<0.001 * * *$ & $<0.001 * * *$ & $<0.001 * * *$ \\
\hline & & LS & $<0.001 * * *$ & $0.001 * *$ & 0.178 \\
\hline & TFDP & LA & $<0.001 * * *$ & $<0.001 * * *$ & $<0.001 * * *$ \\
\hline & & LS & $<0.001 * * *$ & $<0.001 * * *$ & 0.564 \\
\hline & LA & LS & $<0.001 * * *$ & $<0.001 * * *$ & $<0.001 * * *$ \\
\hline \multirow[t]{6}{*}{$2 \mathrm{~B}$} & TFDS & TFDP & $<0.001 * * *$ & $<0.001 * * *$ & 0.991 \\
\hline & & LA & $<0.001 * * *$ & $<0.001 * * *$ & $<0.001 * * *$ \\
\hline & & LS & $<0.001 * * *$ & $<0.001 * * *$ & 0.697 \\
\hline & TFDP & LA & $<0.001 * * *$ & $<0.001 * * *$ & $<0.001 * * *$ \\
\hline & & LS & $<0.001 * * *$ & $<0.001 * * *$ & 0.845 \\
\hline & LA & LS & $<0.001 * * *$ & $<0.001 * * *$ & $<0.001 * * *$ \\
\hline $3 \mathrm{~A}$ & TFDS & TFDP & $<0.001 * * *$ & $<0.001 * * *$ & 0.32 \\
\hline
\end{tabular}

Est=Estructura; EC=Estructura comparada; $\mathrm{AT}$ : área de la sección transversal $\left(\mathrm{cm}^{2}\right)$; LM: ancho latero medial $(\mathrm{cm})$; DP: ancho dorso palmar (cm).

$*=\mathrm{p}<0.05, * *=\mathrm{p}<0.01, * * *=\mathrm{p}<0.001$.

Se encontraron diferencias en los valores de la misma estructura según la zona, las diferencias de los valores de los tendones y ligamentos entre las distintas zonas, como se logra apreciar en la Tabla 3. 
Tabla 3. Comparación (diferencia de medias) de mediciones morfométricas ultrasonográficas entre zonas de los tendones flexores digitales y ligamentos de la región metacarpal palmar en 30 CCC.

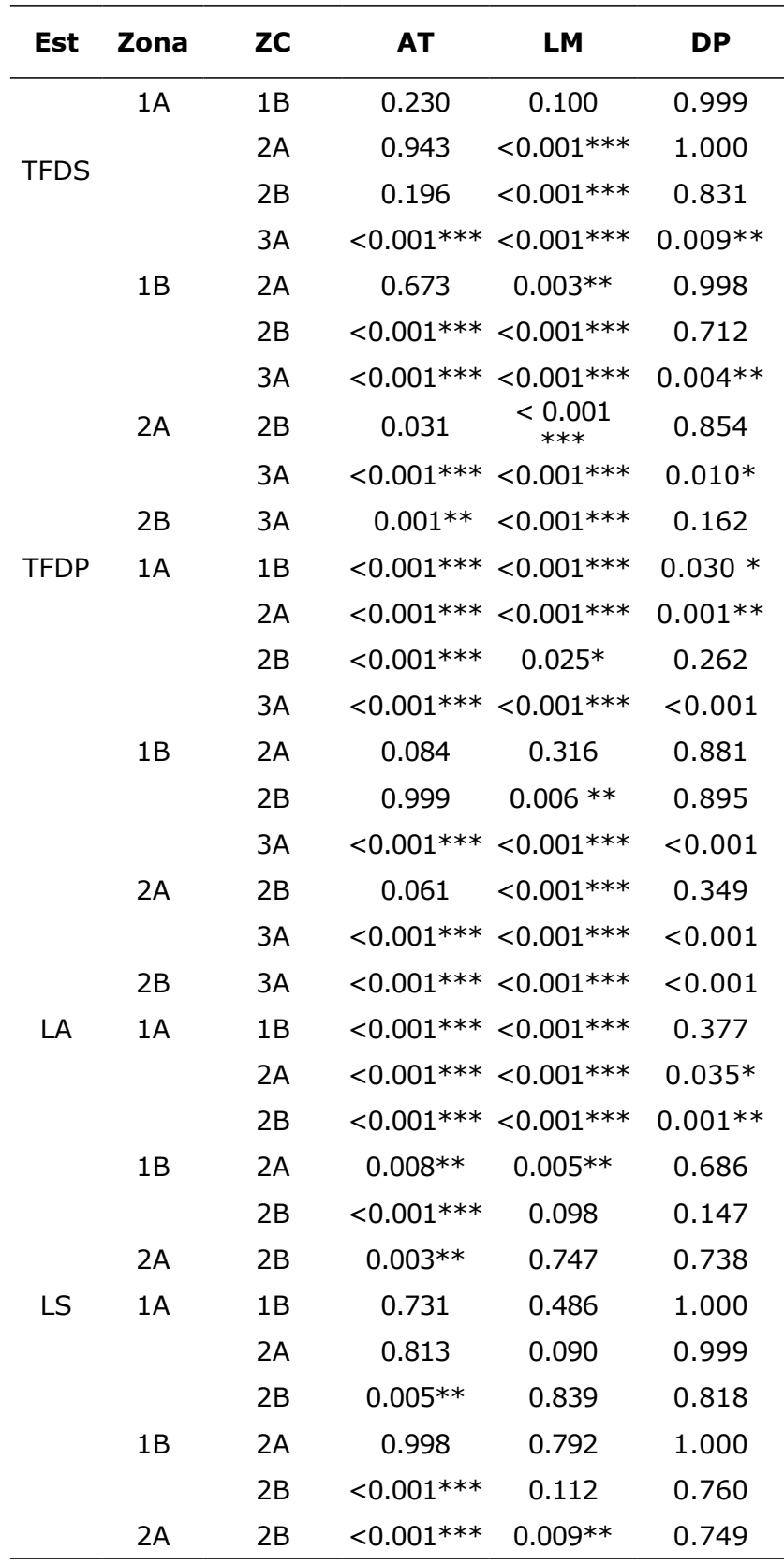

Est $=$ Estructura; $\mathrm{ZC}=$ Zona comparada; $\mathrm{EC}=$ Estructura comparada; AT: área de la sección transversal $\left(\mathrm{cm}^{2}\right)$; LM: ancho latero medial $(\mathrm{cm}) ;$ DP: ancho dorso palmar $(\mathrm{cm})$. $*=\mathrm{p}<0.05, * *=\mathrm{p}<0.01, * * *=\mathrm{p}<0.001$.

Al analizar DP, se encontró que el TFDP fue la estructura que mayores valores tuvo en las zonas más proximales ( $1 A, 1 B$ y $2 A)$, seguido del $L S$ y el TFDS. De igual forma, en las zonas distales las estructuras que mayores valores presentaron fueron las ramas del ligamento suspensorio y vale mencionar que fue el mayor valor de DP de todas las regiones ( $1 \mathrm{~cm}$ en promedio).

Intervalos de confianza. Al determinar que el único factor que influyó significativamente sobre el tamaño de los tendones y ligamentos metacarpales palmares de los 30 CCC fue la zona, en la Tabla 4 se presentan los rangos de referencia como intervalos de confianza del $95 \%$ para cada medida (AT, LM y DP) de cada estructura en las diferentes zonas.

Tabla 4. Intervalos de confianza del $95 \%$ de las mediciones morfométricas ecográficas a 5 zonas de los tendones y ligamentos flexores digitales de la región metacarpal palmar.

\begin{tabular}{|c|c|c|c|c|c|c|c|}
\hline & \multicolumn{3}{|c|}{ Miembro izquierdo } & \multicolumn{3}{|c|}{ Miembro derecho } \\
\hline & & AT & LM & DP & AT & LM & DP \\
\hline \multirow{5}{*}{ TFDS } & $1 \mathrm{~A}$ & $\begin{array}{c}0.484 \\
-0.550\end{array}$ & $\begin{array}{c}1.020 \\
-1.090\end{array}$ & $\begin{array}{c}0.492 \\
-0.545\end{array}$ & $\begin{array}{c}0.475 \\
-0.539\end{array}$ & $\begin{array}{c}1.000 \\
-1.080\end{array}$ & \\
\hline & 1B & $\begin{array}{c}0.469 \\
-0.519\end{array}$ & $\begin{array}{c}1.090 \\
-1.160\end{array}$ & $\begin{array}{c}0.409 \\
-0.455\end{array}$ & $\begin{array}{c}0.439 \\
-0.494\end{array}$ & $\begin{array}{c}1.050 \\
-1.130\end{array}$ & $\begin{array}{c}0.411 \\
-0.442\end{array}$ \\
\hline & $2 \mathrm{~A}$ & $\begin{array}{c}0.470 \\
-0.537\end{array}$ & $\begin{array}{c}1.140 \\
-1.230\end{array}$ & $\begin{array}{c}0.357 \\
-0.398\end{array}$ & $\begin{array}{c}0.466 \\
-0.532\end{array}$ & $\begin{array}{c}1.150 \\
-1.250\end{array}$ & $\begin{array}{c}0.351 \\
-0.392\end{array}$ \\
\hline & $2 B$ & $\begin{array}{c}0.522- \\
0.591\end{array}$ & $\begin{array}{c}1.290 \\
-1.380\end{array}$ & $\begin{array}{c}0.342 \\
-0.387\end{array}$ & $\begin{array}{c}0.503 \\
-0.569\end{array}$ & $\begin{array}{c}1.290 \\
-1.400\end{array}$ & $\begin{array}{c}0.325 \\
-0.357\end{array}$ \\
\hline & $3 \mathrm{~A}$ & $\begin{array}{c}0.571 \\
-0.634\end{array}$ & $\begin{array}{c}1.630 \\
-1.750 \\
\end{array}$ & $\begin{array}{c}0.279 \\
-0.306\end{array}$ & $\begin{array}{c}0.574 \\
-0.644 \\
\end{array}$ & $\begin{array}{c}1.580 \\
-1.740 \\
\end{array}$ & $\begin{array}{c}0.279 \\
-0.313\end{array}$ \\
\hline \multirow{5}{*}{ TFDP } & $1 \mathrm{~A}$ & & & & & & \\
\hline & 1B & $\begin{array}{c}0.586 \\
-0.648\end{array}$ & & & & & \\
\hline & $2 \mathrm{~A}$ & $\begin{array}{c}0.556 \\
-0.595\end{array}$ & $\begin{array}{c}0.876 \\
-0.932\end{array}$ & $\begin{array}{c}0.678 \\
-0.725\end{array}$ & $\begin{array}{c}0.567 \\
-0.618\end{array}$ & $\begin{array}{c}0.887 \\
-0.948\end{array}$ & $\begin{array}{c}0.700 \\
-0.756\end{array}$ \\
\hline & 2B & $\begin{array}{c}0.594 \\
-0.658\end{array}$ & & & & & \\
\hline & $3 A$ & & & & & & \\
\hline \multirow{4}{*}{ LA } & $1 \mathrm{~A}$ & $\begin{array}{c}0.493 \\
-0.576\end{array}$ & $\begin{array}{c}1.010 \\
-1.100\end{array}$ & $\begin{array}{c}0.404 \\
-0.468\end{array}$ & $\begin{array}{c}0.460 \\
-0.537\end{array}$ & $\begin{array}{c}0.977 \\
-1.070\end{array}$ & $\begin{array}{c}0.381 \\
-0.448\end{array}$ \\
\hline & 1B & $\begin{array}{c}0.393 \\
-0.454\end{array}$ & $\begin{array}{c}0.844 \\
-0.926\end{array}$ & $\begin{array}{c}0.368 \\
-0.412\end{array}$ & $\begin{array}{c}0.394 \\
-0.463\end{array}$ & $\begin{array}{c}0.849 \\
-0.949\end{array}$ & $\begin{array}{c}0.365 \\
-0.418\end{array}$ \\
\hline & $2 \mathrm{~A}$ & $\begin{array}{c}0.348 \\
-0.410\end{array}$ & $\begin{array}{c}0.771 \\
-0.865\end{array}$ & $\begin{array}{c}0.340 \\
-0.391\end{array}$ & $\begin{array}{c}0.336 \\
-0.405\end{array}$ & $\begin{array}{c}0.789 \\
-0.867\end{array}$ & $\begin{array}{c}0.322 \\
-0.387\end{array}$ \\
\hline & 2B & $\begin{array}{c}0.304 \\
-0.353\end{array}$ & $\begin{array}{c}0.804 \\
-0.881\end{array}$ & $\begin{array}{c}0.287 \\
-0.320\end{array}$ & $\begin{array}{c}0.286 \\
-0.333\end{array}$ & $\begin{array}{c}0.809 \\
-0.880\end{array}$ & $\begin{array}{c}0.257 \\
-0.297\end{array}$ \\
\hline \multirow{4}{*}{ LS } & & $\begin{array}{c}0.790 \\
-0.890\end{array}$ & $\begin{array}{c}1.130 \\
-1.210\end{array}$ & $\begin{array}{c}0.680 \\
-0.731\end{array}$ & $\begin{array}{c}0.821 \\
-0.921\end{array}$ & $\begin{array}{c}1.130 \\
-1.210\end{array}$ & $\begin{array}{c}0.702 \\
-0.750\end{array}$ \\
\hline & & $\begin{array}{c}0.782 \\
-0.869\end{array}$ & $\begin{array}{c}1.100 \\
-1.170\end{array}$ & $\begin{array}{c}0.702 \\
-0.742\end{array}$ & $\begin{array}{c}0.790 \\
-0.879\end{array}$ & $\begin{array}{c}1.100 \\
-1.190\end{array}$ & $\begin{array}{c}0.705 \\
-0.745\end{array}$ \\
\hline & $2 \mathrm{~A}$ & $\begin{array}{c}0.776 \\
-0.864\end{array}$ & $\begin{array}{c}1.060 \\
-1.150\end{array}$ & $\begin{array}{c}0.697 \\
-0.745\end{array}$ & $\begin{array}{c}0.804 \\
-0.891\end{array}$ & $\begin{array}{c}1.090 \\
-1.180\end{array}$ & $\begin{array}{c}0.711 \\
-0.758\end{array}$ \\
\hline & $2 B$ & $\begin{array}{c}0.889 \\
-1.030 \\
\end{array}$ & $\begin{array}{c}1.130 \\
-1.240 \\
\end{array}$ & $\begin{array}{c}0.727 \\
-0.833\end{array}$ & $\begin{array}{c}0.860 \\
-0.972\end{array}$ & $\begin{array}{c}1.150 \\
-1.240 \\
\end{array}$ & $\begin{array}{c}0.658 \\
-0.775\end{array}$ \\
\hline RM & $3 A$ & $\begin{array}{c}0.582 \\
-0.679 \\
\end{array}$ & $\begin{array}{c}0.610 \\
-0.667 \\
\end{array}$ & $\begin{array}{c}0.965 \\
-1.060 \\
\end{array}$ & $\begin{array}{c}0.582 \\
-0.678 \\
\end{array}$ & $\begin{array}{c}0.596 \\
-0.646 \\
\end{array}$ & $\begin{array}{c}0.983 \\
-1.070 \\
\end{array}$ \\
\hline RL & $3 A$ & $\begin{array}{c}0.592 \\
-0.711 \\
\end{array}$ & $\begin{array}{c}0.613 \\
-0.676\end{array}$ & $\begin{array}{c}0.984 \\
-1.070\end{array}$ & $\begin{array}{c}0.629 \\
-0.696\end{array}$ & $\begin{array}{c}0.636 \\
-0.673 \\
\end{array}$ & $\begin{array}{c}1.010 \\
-1.070\end{array}$ \\
\hline
\end{tabular}

TFDS: Tendón flexor digital superficial; TFDP: tendón flexor digital profundo; LA: ligamento accesorio; LS: ligamento suspensorio; RM: rama medial del ligamento suspensorio; RL: rama lateral del ligamento suspensorio; AT: área de la sección transversal, LM: ancho latero medial, DP: ancho dorso palmar 


\section{DISCUSION}

En caballos islandeses, Boehart et al (7) reportó que el ligamento suspensorio mostró los mayores valores para el área transversal en las tres primeras zonas, seguido del tendón flexor digital profunda acompañado de su ligamento accesorio y por último el tendón flexor digital superficial. En los caballos criollos colombianos de este estudio, el comportamiento de esta medida fue similar; el ligamento suspensorio fue la estructura de mayor área en dichas zonas, seguido del TFDP, pero en este caso el TFDS obtuvo mayores valores que el ligamento accesorio del TFDP.

Kasashima et al (14) reportaron que no existe diferencia significativa en el área transversal del TFDS entre el miembro anterior izquierdo y derecho en potros PSI en crecimiento bajo un régimen de ejercicio al igual que los que no se encontraban realizando ejercicio. Esto concuerda con lo encontrado en el presente estudio, donde no existió diferencia entre ambos miembros para dicha estructura.

Avella et al (15) en caballos de carrera nacional reportaron que existió diferencia significativa $(p=0.007)$, al comparar el área transversal del TFDS de la región 2B entre machos y hembras siendo en hembras $8 \mathrm{~mm}^{2}$ menor que en machos, esto concuerda con lo encontrado en el presente estudio ya que en estos animales también existió diferencia significativa entre machos y hembras. Existen diversos factores que podrían influir en este resultado como el largo de la cuartilla, la conformación de los cascos por el estrés que este puede producir en los tendones flexores o incluso la diferencia entre la intensidad del entrenamiento entre machos y hembras, no obstante, es un punto para investigar a mayor profundidad.

Se reportó en yeguas Haflingert (6) que según la edad existieron algunas diferencias significativas en los valores estudiados como en el caso del DP de la zona $1 \mathrm{~B}$ y $3 \mathrm{~B}$ del tendón flexor digital superficial principalmente, donde fue significativamente mayor esta medida en animales menores a 7 años, y de la misma manera mayor en el caso del flexor digital profundo en los animales mayores a 7 años. El LM del ligamento accesorio del TFDP, tuvo mayores valores en la zona $1 \mathrm{~B}$ de animales menores a 7 años. Lo cual difiere del CCC, donde no existió una diferencia significativa para las variables en relación a la edad.
Celimli et al (11) reportaron que el área del tendón flexor digital superficial en la zona $1 \mathrm{~A}$ es de 0.62 $\mathrm{cm}^{2}$ para caballos árabes, dicho valor es mayor al encontrado en caballos criollos colombianos $\left(0.51 \mathrm{~cm}^{2}\right)$. En la misma zona, Smith et al (4) reportaron que dicho valor es de $1.28 \mathrm{~cm}^{2}$, siendo más del doble de lo encontrado en el CCC. En la mayoría del resto de las zonas evaluadas, se pudo observar que esta relación entre las medidas de las estructuras para estas razas se conserva. Por lo tanto, allí deriva la importancia de conocer los valores propios de cada estructura. Finalmente se puede afirmar que dichos valores son específicos para cada raza.

Son escasos en la literatura los estudios que evalúan la relación en el tamaño de los tendones y el índice de masa corporal, no obstante, Boehart et al (6), en yeguas haflingert reportó que existe diferencia significativa entre el LM del TFDS en la zona $1 \mathrm{~A}(\mathrm{p}=0.04)$ y $1 \mathrm{~B}(\mathrm{p}=0.04) \mathrm{de}$ acuerdo con el IMC, siendo mayor en animales con un IMC igual o menor a $186.99 \mathrm{~kg} / \mathrm{m}^{2}$. De igual forma, para el TFDP en la zona $1 \mathrm{~B}$ donde se encontró que existió diferencia significativa $p=$ 0.032 para el LM, siendo mayor en los animales de un IMC menor o igual a $186.99 \mathrm{~kg} / \mathrm{m}^{2}$. Esto difiere de los animales del presente estudio donde no se encontró ninguna diferencia cuando se comparó el AT, DP y LM entre los grupos formados de acuerdo al IMC, lo que significa que la influencia de esta variable en las medidas morfométricas de los tendones y ligamentos metacarpales palmares varía según la raza y podría estar justificado en que un animal más magro seguramente ejercerá un estrés biomecánico diferente sobre una estructura en comparación con un animal más graso.

En caballos islandeses y en yeguas haflingert, Boehart et al $(6,7)$ reportaron que al igual que lo encontrado en los caballos de este estudio existió diferencia significativa entre los valores del mismo tendón o ligamento entre las diferentes zonas. Esto es de esperarse debido a los grandes cambios que sufren las estructuras a lo largo de su recorrido. Por lo tanto, es necesario aclarar que no tiene el mismo valor diagnostico la medida de una estructura en las diferentes zonas y por obligación todas las estructuras deberán ser evaluadas por completo en las diferentes zonas de la región metacarpal palmar teniendo en cuenta sus características anatómicas e histológicas para la interpretación de la imagen ecográfica. 
Reyes et al - Tendones metacárpales de caballos criollos colombianos.

En conclusión, el ligamento suspensorio y el TFDP fueron las estructuras de mayor tamaño en las zonas proximales, así como en las zonas distales. No existe diferencia significativa entre miembros y tampoco se encontró relación entre el IMC y las medidas morfométricas para estas estructuras. En cada región los valores varían de acuerdo con su recorrido anatómico y es importante tener en cuenta la zona y estructura que se está evaluando para poder hacer una correcta interpretación. Estos valores son de mucha importancia en la práctica de la medicina veterinaria ya que son medidas específicas del caballo criollo colombiano y permitirán un mejor desarrollo de la práctica veterinaria en esta raza.

\section{Conflicto de intereses}

Los autores declaramos que no existen conflictos de intereses en esta publicación.

\section{Agradecimientos}

Los autores agradecen a la Universidad de Córdoba y a los diferentes criaderos. Adicionalmente, el primer autor agradece a los médicos veterinarios Mateo Velásquez, Juan Pablo Martínez e Iván Avalos por sus aportes profesionales.

\section{REFERENCIAS}

1. Rantanen NW. The use of diagnostic ultrasound in limb disorders of the horse: a preliminary report. J Equine Vet Sci. 1982; 2(2):62-64. https://doi.org/10.1016/ S0737-0806(82)80021-X

2. Agut $A$, Martinez $M$, Sanchez $A$, Soler $M$, Rodriguez M. Ultrasonographic characteristics (cross-sectional area and relative echogenicity) of the digital flexor tendons and ligaments of the metacarpal region in Purebred Spanish horses. Vet J. 2009; 180(3):377-383. https://doi. org/10.1016/j.tvjl.2008.01.012

3. Van schie $H$, Bakker $E$, Jonker $M$, Van weeren P. Ultrasonographic tissue characterization of equine superficial digital flexor tendons by means of gray level statistics. AJVR. 2000; 61(2):210-219. https://doi.org/10.2460/ ajvr.2000.61.210

4. Smith $\mathrm{R}$, Jones $\mathrm{R}$, Webbon P. The crosssectional areas of normal equine digital flexortendons determined ultrasonog raphicaly. Equine Vet J. 1994; 26(6):460-465. https://doi.org/10.1111/j.2042-3306.1994. tb04050.x
5. Cuesta I, Riber C, Pinedo M, Gata J, Castejon F. Ultrasonographic measurement of palmar metacarpal tendon and ligament structures in the horse. Vet Radiol Ultrasound. 1995; 36(2):131-136. https://doi. org/10.1111/j.1740-8261.1995.tb00232.x

6. Boehart S, Arndt G, Carstanjen B. Ultrasonographic morphometric measurements of digital flexor tendons and ligaments of the palmar metacarpal region in haflinger horses. Anat. Histol. Embryol. 2010; 39(4):366-375. https://doi. org/10.1111/j.1439-0264.2010.01003.x

7. Boehart S, Arndt G, Rindermann G, Gmachl M, Carstanjen B. Assessment of ultrasonographic morphometric measurements of digital flexor tendons and ligaments of the palmar metacarpal region in Icelandic Horses. AJVR. 2010; 71(12):1425-1431. https:// doi.org/10.2460/ajvr.71.12.1425

8. Cardona J, Reyes B, Martínez M. Cronometría dentaria en equinos. Primera edición. Fondo editorial Universidad de Córdoba. 2019. https://repositorio.unicordoba.edu.co/ handle/ucordoba/2204 
9. Docking S, Daffy J, Van Schie H, Cook J. Tendon structure changes after maximal exercise in the Thoroughbred horse: Use of ultrasound tissue characterization to detect in vivo tendon response. Vet J. 2012; 194(3):339-342. https://doi.org/10.1016/j. tvjl.2012.04.024

10. Cardona J, Reyes B, Martínez M. Semiología y propedéutica clínica del aparato locomotor en grandes animales. Primera edición. Fondo editorial Universidad de Córdoba: Colombia; 2019. https://repositorio.unicordoba.edu. co/handle/ucordoba/2203

11. Çelimli N, Seyrek D, Kaya $M$. Morphometric measurements of flexor tendons and ligaments in Arabian horses by ultrasonographic examination and comparison with other breeds. Equine Vet Educ. 2004; 16(2):81-85. https://doi. org/10.1111/j.2042-3292.2004.tb00271.x

12. Pickersgill C, Marr C, Reid S. Repeatability of diagnostic ultrasonography in the assessment of the equine superficial digital flexor tendon. Equine Vet J. 2001; 33(1):33-37. https:// doi.org/10.2746/042516401776767494
13. Whitcomb M. Ultrasonographic evaluation of the distal extremity. J. Equine Vet. Scie. 2009; 29(1):47-59. http://dx.doi. org/10.1016/j.jevs.2008.11.010

14. Kasashima $Y$, Smith $R$, Birch $H$, Takahashi T, Kusano K, Goodship A. Exerciseinduced tendon hypertrophy: crosssectional area changes during growth are influenced by exercise. Equine Vet. J. 2002; 34(S34):264-268. https://doi. org/10.1111/j.2042-3306.2002.tb05430.x

15. Avella C, Ely E, Verheyen K, Price J, Wood J, Smith R. Ultrasonographic assessment of the superficial digital flexor tendons of National Hunt racehorses in training over two racing seasons. Equine Vet J. 2009; 41(5):449-454. https://doi. org/10.2746/042516409×3910 